\title{
End-to-end adaptation scheme for ubiquitous remote experimentation
}

\author{
Christophe Salzmann · Denis Gillet • \\ Philippe Mullhaupt
}

Received: 4 December 2006/Accepted: 8 May 2007/Published online: 30 October 2007

(C) Springer-Verlag London Limited 2007

\begin{abstract}
Remote experimentation is an effective e-learning paradigm for supporting hands-on education using laboratory equipment at distance. The current trend is to enable remote experimentation in mobile and ubiquitous learning. In such a context, the remote experimentation software should enable effective telemonitoring and teleoperation, no matter the kind of device used to access the equipment. It should also be sufficiently lenient so as to handle the rapidly evolving wireless and mobile communication environment. While the current Internet bandwidth allows remote experimentation to work flawlessly on fixed connections such as LANs, mobile users suffer from both the versatile nature of wireless communications and the limitation of the mobile devices. These conditions impose that the remote experimentation software should integrate adaptation features. For effective ubiquitous remote experimentation, it should ideally be guaranteed that the information representing the state of the remote equipment is rendered (to the end user) at the same pace at which it has been acquired, yet possibly at the cost of a somewhat minimal time delay between the acquisition and rendering phases. In this respect, an end-to-end adaptation scheme is proposed that explicitly handles the inherent variability of the connection and the versatility of the mobile devices considered in ubiquitous remote experimentation. Instead of relying on a stochastic approach, the proposed adaptation scheme relies on a deterministic mass-balance equivalence model. The effectiveness of the proposed
\end{abstract}

C. Salzmann $(\varangle) \cdot$ D. Gillet $\cdot$ P. Mullhaupt

Laboratoire d'automatique,

Ecole Polytechnique

Fédérale de Lausanne, Lausanne, Switzerland

e-mail: christophe.salzmann@epfl.ch adaptation scheme is demonstrated in critical conditions corresponding to remote experimentation carried out using a PDA over a Bluetooth link.

Keywords End-to-end adaptation ·

Remote experimentation - Wireless communication

\section{Introduction and context}

Remote experimentation is a specific case of real-time interaction for which the interaction is not carried out between two human beings (as it is the case in Videoconferencing or Voice-over-IP) but between a human being (at the client side) and a physical equipment (at the server side, see Fig. 1).

Remote experimentation is typically introduced to complement hands-on laboratory sessions in higher education [1] and to avoid traveling to training centers in distance learning. Robotic and mechatronic systems are often remotely controlled through the Internet [2-5] as they exhibit visually observable dynamical behaviors. In addition, comparison between simulation and actual implementation results is an important element of the educational methodology in robotics, mechatronics and control [6]. As a consequence and without loss of generality, this paper focuses on ubiquitous remote experimentation on mechatronics systems in control education.

The different control design and implementation steps taught to students in control courses (system identification, controller design, performance validation, etc.) can be efficiently carried out remotely on mechatronic systems provided that special care is taken on key issues. The most challenging issue with mechatronic systems is the pace at which the information needs to be updated so that the distant 


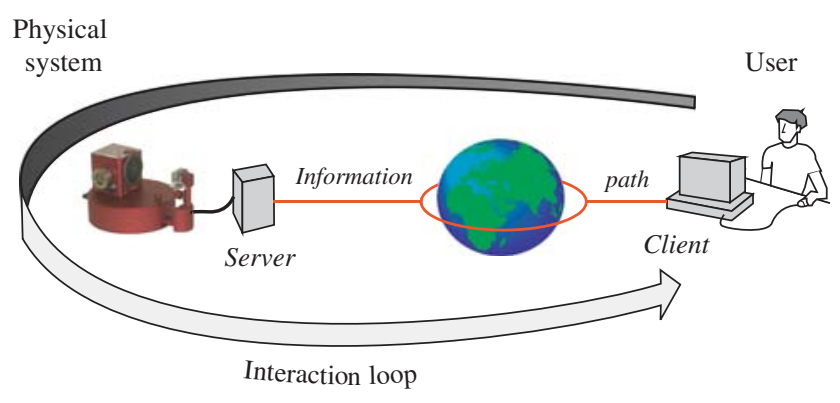

Fig. 1 Typical remote experimentation configuration for the control of phyiscal system

user perceives correctly the dynamics of the equipment (i.e. the displacement of the moving parts). Compared to other systems that may require offline analysis such as chemical systems, many real-time measurements (such as position, speed, acceleration, etc.) are easily available on mechatronic systems. This information is to be played back at the client side in a timely manner so as to help the student to soundly perceive the physical equipment and its dynamics. The information representing the state of the distant equipment is generally available in the form of a video stream and an oscilloscope window displaying the measurement data acquired on the equipment. Additional synthetic representation combining two sources of information such as an augmented reality can also be provided. It implies tight time synchronization between the different streams of information. Measurement data acquired on the equipment can also be interpreted and rendered using other means, for instance force feedback or audio signal. Notice that the most challenging aspect of mechatronic systems is the intrinsic time constant of the phenomenon to be observed that is generally of the same order of magnitude as the transmission time in remote experimentation. The particular nature of the physical equipment, as opposed to a human being, imposes specific constrains and requires dedicated solutions [7, 8]. This paper focuses on ubiquitous remote experimentation solutions that have strong real-time constraints due to both the transmission channel characteristics and versatility, as well as the physical equipment dynamics.

The objective of a ubiquitous remote experimentation solution is to make the student interaction with the distant system as close as possible to the actual work on the real equipment. In other words, the best possible feedback to a user action has to be provided such that it limits the inherent drawbacks stemming from the distance separating a user at the client side from the operated physical equipment at the server side (Fig. 1).

This distance generates two undesirable effects, namely (1) the transmission delay for the information to travel from one end to the other and back, and (2) the difficulty to reproduce (at the client side) the state of the distant equipment together with its dynamics and operating conditions.

An efficient remote experimentation solution should not only maximize the user experience, but also adapt, in realtime, to the underlying end-to-end infrastructure. This should be undertaken by adapting the information transmission to the characteristics of the various components found along the information path, including not only the end-to-end transmission link but also the client and the server considered at the application level.

Working at the application level allows taking advantage of the knowledge concerning the semantic content of the transmitted information. The underlying levels such as the transmission one (i.e. TCP) only deal with bytes of data and thus may not behave appropriately when, for example, some information has to be discarded.

In order to be available on a wide range of applications and platforms, ubiquitous remote experimentation solutions should be implemented using available technologies. They should also adapt and take advantage of new standards while following the Internet best practice $[9,10]$.

While it can be assumed that the server application/ equipment is known before hand, this is not true for the client application/device. Thus, relevant technology and protocols that are available at the client side are to be selected to transmit information. As a consequence, the adaptation scheme has to be independent of the underlying protocols and infrastructure used to acquire, transfer and play back the information.

Therefore, a novel end-to-end adaptation scheme is proposed that satisfy the above ubiquitous remote-experimentation requirements to ensure that the distant user gets the best possible feedback given both the available infrastructure and the working conditions.

The paper is organized as follows. First, in Sect. 2, the user requirements in ubiquitous remote experimentation are asserted through a dedicated definition of Quality of Service (QoS). Then, in Sect. 3, the end-to-end abstractions of the considered infrastructure observed at the application level is presented. It includes the communication link together with both the server and the client devices. Section 4 describes the metrics used to compensate the lack of a direct sensor for estimating the defined QoS. Section 5 presents the end-toend adaptation scheme used to enforce the given QoS. The validation of this scheme is finally presented in Sect. 6 together with a real-world mobile setup introduced for hands-on experimentation in engineering education.

\section{Quality of Service for remote experimentation}

The QoS defines the level of satisfaction, usability or efficiency for a given service, requirement or solution. The 
QoS is highly dependent on the application context it is defined for. Different applications have different requirements that translate into different definitions for the QoS $[11,12]$. For example, at the network transmission level, throughput and latency would define the QoS for Web surfing, while the packet jitter and packet delay would be relevant for voice (VoIP) transmission [13].

The QoS can be defined at various levels [14]. For example, at the end-user level the QoS maps some subjective features like immersion, presence and comfort to measurable values. At the physical layer level, the QoS describes characteristics such as the bit error rate for a transmission link [15]. At the application level, the Web service QoS requirements define availability, accessibility, integrity, performance (measured in terms of throughput and latency), reliability, regularity and security [16]. This differs from the above QoS defined for Web performances at the network level. Network-centric and end-system centric QoS definitions for video delivery over wireless Internet are presented in [17].

The QoS is often confused or amalgamated with the QoS enforcement mechanisms. The QoS enforcement mechanisms are numerous. Each QoS specification may lead to many enforcement mechanisms.

Remote experimentation requires a specific QoS to characterize the suitability of the feedback provided to the distant user. Three key conditions that need to be satisfied at the application level so as to provide a suitable user experience in the context of hands-on engineering education were identified through laboratory sessions analysis and users surveys [18-20]. These conditions are: (1) the level of interaction which represents how quickly a feedback is provided to the user should be high enough; (2) the system dynamic rendering which represents how accurately in time the behavior of the remote system is perceived should be accurate enough; (3) the amount of semantic content that represents how well the distant equipment state and conditions of operation can be perceived by the client should be rich enough. The three conditions are now detailed.

The level of interaction can be characterized by the delay observed between the time at which an action is performed and the time at which its effect can be perceived by the user. The delay represents the information round-trip time measured at the application level. This delay is a function of many factors, especially buffers that are found along the path taken by the information. For a valuable user experience, this delay should be as small as possible; small being defined in accordance with the dynamics of the distant equipment. This constraint is one of the main differences between remote experimentation and other realtime applications such as video-streaming. Human eyes will notice a delay larger than $50 \mathrm{~ms}$ [21], similarly a delay larger than $200 \mathrm{~ms}$ is noticeable in audio transmission [22]. While these values express minimum values, a delay smaller than a second is noticeable by the user, albeit acceptable since it is short enough to avoid any inappropriate sensorimotor reaction from the user [23].

The dynamics of the distant system needs to be perceived at the client side. This implies that both the acquisition and the rendering paces should be adequate and equal. If the pace at which the server acquires the information is not adequate, the user might get a biased or wrong perception of the actual dynamical behavior of the distant system. Similarly, the pace at which the information is perceived by the user at the client side should be adequate (a wheel rotation at a constant speed should be perceived as such remotely). The rendering pace can be altered by the jitter introduced by the non-deterministic nature of the transmitting channel (i.e. the wired or wireless Internet). In multimedia applications, buffering techniques are traditionally used at the client side to smooth the jitter when playing the information back [24]. Such buffers add delay to the transmission and therefore should not be considered since they depreciate the user perception level necessary for effective remote experimentation.

The semantic content has to be rich enough to allow the perception of the state and the conditions of operation. There are various sources of information that can be used to provide this information. Video streams, images, sounds, force-feedback, virtual-reality representation or data history displayed in an oscilloscope-like window can be used for that purpose. For a given type of representation, however, the higher the quality of the sent information, the better the perception of the state is. For instance, a good quality color picture, bigger in size, is more informative than a low quality, black and white image, smaller in size. Measuring the quality of the perception automatically is a very challenging operation [25-27]. Therefore, we assume from now on that the quantity (size) of transmitted information reflects the quality of the semantic content that needs to be maximized.

\section{End-to-end model}

The objective of the end-to-end model is to describe the remote-experimentation underlying infrastructure in a suitable manner. It should be sufficiently accurate for implementing the end-to-end adaptation scheme. Traditionally, end-to-end (E2E) approaches used to ensure fairness in the transmission by only considering the network path between the two hosts [28]. The end-to-end infrastructure, which includes (to some extent) the client and the server applications, has also been considered by others [29]. An end-to-end infrastructure that incorporates 
both the coder/decoder (for video streaming) and an endto-end buffer-management mechanism is presented in [30]. Similarly, [31] proposed a variable frame-rate encoding for video streaming. The authors in [17] consider the end-toend structure over wireless link for video transmission.

Our proposed adaptation scheme for remote experimentation considers the infrastructure as a whole. It encompasses both the client and the server applications/ devices. This guarantees that the QoS characterization correctly reflects the whole infrastructure ability (or inability) to handle the flow of transmitted information. Nowadays and especially in an ubiquitous context, the client application/device may be the limiting factor in the information transmission chain. Therefore, it needs to be properly considered [32]. For example, a PDA device may not be able to handle (decode/render) more than 4 images per second, while the server may deliver 10 frames per second over the connection link. Also, on the same device, it is possible to consider two applications performing similar tasks but written in different languages, one compiled and the other interpreted, which results in a significant difference when comparing the execution time. Similarly, at the server side, the information encoding using temporal compression such as in MPEG, where more than one sample of information is required, may add delay that needs to be considered [33]. For example, the encoding of a 15 frame per second video stream with a temporal compression that needs to process 5 frames at a time adds a delay of $1 / 3$ of a second, which is in the same order of magnitude as the transport delay for a transatlantic Internet connection (ping typically shows about 20 hops).

The proposed representation of the E2E information transmission path includes three components abstracted from the various elements found at the client, the server and the network levels. The transmitted information is also abstracted in semantic units called blocks as defined in Sect. 3.2. The abstraction is necessary to build a model which is independent of the actual implementation of the various elements but which capture the relevant features of the underlying versatile infrastructure.

\subsection{Components abstraction}

The E2E physical infrastructure (Fig. 2a) is abstracted by the E2E virtual layer (Fig. 2b). The path is considered from the information capture, at the server side, to the information restitution, at the client side. The E2E information transmission goes through three main steps, namely (1) the information acquisition and encoding, (2) the transmission of the information over the network, and (3) the decoding and the rendering at the other end.

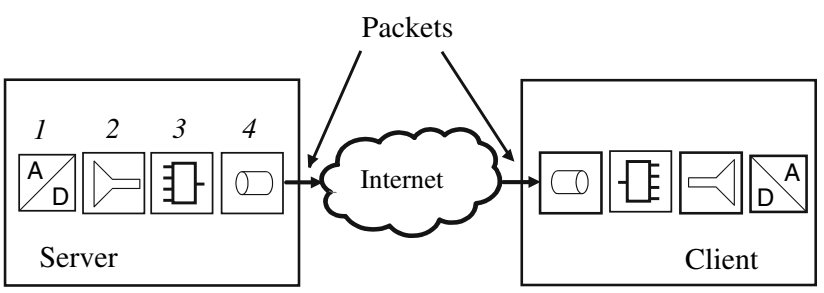

End-to-end physical infrastructure

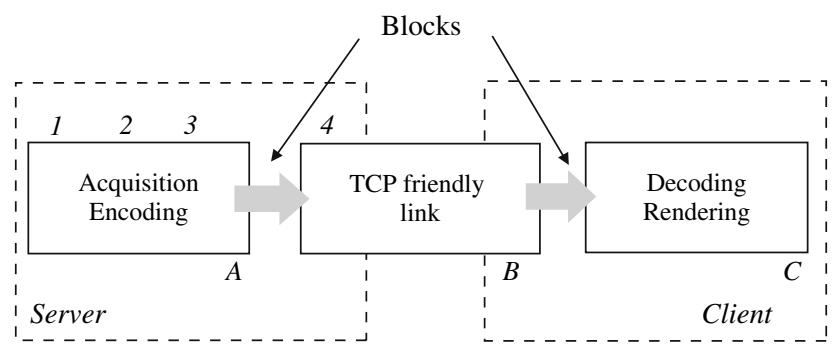

End-to-end virtual layer

Fig. 2 a The end-to-end physical infrastructure. $1 \mathrm{~A} / \mathrm{D}$ conversion, 2 compression, 3 multiplexing, 4 transmission. b The E2E virtual layer or E2E infrastructure

The acquisition-and-encoding component (Fig. 2b-A) functionality consists in transforming the acquired measurements (data, video, etc.), representing the state of the real equipment and its conditions of operation, to a digital representation. This should follow a format that is suitable for transmission. The decoding-and-rendering component (Fig. 2b-C) is similar to the acquisition-and-encoding component, with the difference that the information processing is carried out in reverse order. The transmission component (Fig. 2b-B) encompasses both the server and the client network interfaces. The transmitted information is handled by this component as soon as the control of the transmitted data is transferred from the server application to the underlying OS, i.e. the transmission component is seen through the network APIs. The transmitted information leaves this component as soon as the client component can access it. However, there is no handle to control the transmission over the Internet once the data leaves the computer until it is received at the other end. This is partly due to the non-deterministic aspect of the best-effort Internet network and also to the nature of the protocol used to transmit the data. In other words, neither the network bandwidth nor the network latency can be guaranteed.

\subsection{Block abstraction}

A block encodes the aggregated, necessary and sufficient information that represents the short-term state history, and the operating conditions of the distant system at a given time. 


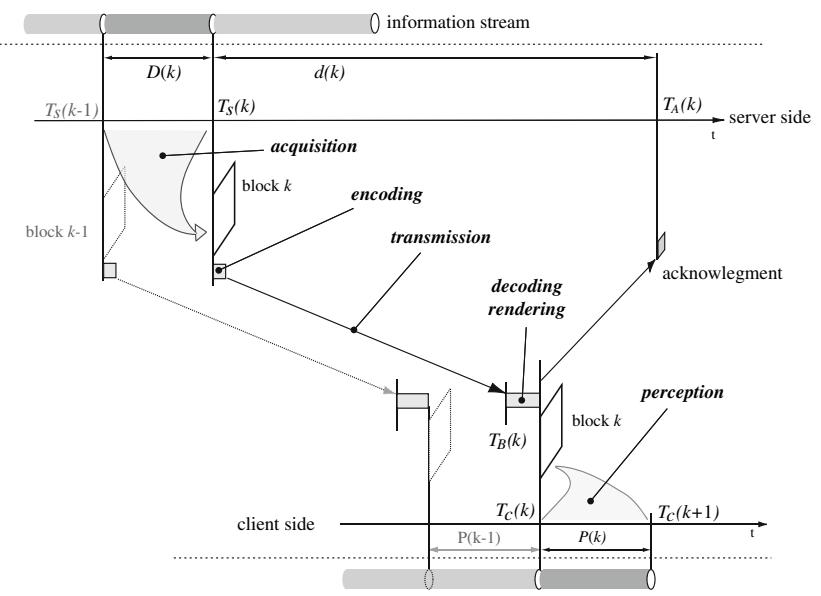

Fig. 3 Block time instants definitions

A block of index $k$ is similar to a snapshot that captures the information regarding the physical system at a given time $T_{\mathrm{S}}(k)$ (Fig. 3). A block is the basic unit of information of size $S(k)$ and duration $D(k)$ having a semantic meaning for the user. For example, a block could be made of a video image combined with measurements acquired concurrently. The successive blocks form the information stream. A block is not to be confused with packets, which are smaller data units transmitted over the network and handled by the network devices (routers) and by the communication protocols.

Once created, the block is processed (encoded) and passed on to the transmission component for its transfer to the client component. The block transmission, from the server to the client, is performed within the transmission component. At time $T_{\mathrm{B}}(k)$, the block $k$ starts to be processed (decoded and rendered) by the client component. Then, at time $T_{\mathrm{C}}(k)$, the block $k$ is presented to the user. At the same time $T_{\mathrm{C}}(k)$, the user perception measurements of the block $k-1$ are performed and sent back to the server. This acknowledgment is processed by the server at the time $T_{\mathrm{A}}(k)$. The block round-trip time measured at the application level $d(k)$ is the time difference between $T_{\mathrm{A}}(k)$ and $T_{\mathrm{S}}(k)$. The information contained in the block $k$ is perceived by the user from the time $T_{\mathrm{C}}(k)$ for a duration $P(k)$. The time interval inbetween block perception at the client side $P(k)$ should be equal to the time interval in-between block creation at the server side $D(k)$. If this is not the case, either the client component or the sever component (or even both) should modify their behavior so as to compensate for this variation.

During the transmission over the Internet, the block might be split into packets.

\section{Metrics for measuring the QoS for remote experimentation}

There is no direct sensor for the proposed QoS. Thus, metrics need to be defined to measure the QoS using the available information. Measurements relative to blocks performed at both the client and the server sides provide the needed information to define metrics that reflect the E2E infrastructure ability to handle the flow of information. Metrics are estimated at the application level in order to capture the characteristics of the whole E2E infrastructure. The three proposed metrics detailed in the next sections are:

(1) $\tau(k)$ for the block periods ratio

(2) $\varepsilon(k)$ for the block sizes ratio

(3) $d(k)$ for the E2E round-trip time

\subsection{Block round-trip time, $d$ metrics}

The block round-trip time $d(k)$ (related to the block $k$ ) represents the time taken for this block to be successfully acquired, transmitted, rendered at the client side, and then acknowledged at the server side. The user perception time $P(k)$ for the block $k$ is not part of the block round-trip time (Fig. 3).

The block round-trip time can be written as

$d(k)=T_{\mathrm{A}}(k)-T_{\mathrm{S}}(k)$

The $d$ metrics ranges from 0 to infinity, $d$ should be as small as possible. Values smaller than $1 \mathrm{~s}$ provide an adequate feedback to the user and can be expected from today's remote experimentation solutions. With $d>1 \mathrm{~s}$, the delay becomes annoying from a sensorimotor point of view. Users may consider that their request has not been properly handled and repeat their initial action.

The level of interaction can be directly measured by the block round-trip time $d$. This measurement takes into account not only the time for the block to transit from the server to the client but also the processing time at both the client and the server applications. Many factors can increase the block round-trip time. Some are controllable, such as the buffers at the server and the client side; some others, like the intrinsic Internet connection round-trip time, cannot be controlled.

Block acknowledgments, measured at the application level, are different from network packets acknowledgments, measured at the network layer level.

In the forthcoming definitions, processing and transmission times are referenced as durations instead of periods 
(periods usually mean regularity) as they are supposed to be modified continuously through the adaptation mechanism.

\subsection{Block duration ratio, $\tau$ metrics}

The block-duration ratio $\tau(k)$ related to the block $k$ is the ratio between the duration of the block perception $P(k)$ measured at the client side and the block duration $D(k)$ defined at the server side (Fig. 3).

The block duration ratio can be written as

$\tau(k)=\frac{D(k)}{P(k)}$

The $\tau$ metrics indicates whether the pace (at which the blocks are created) can be successfully handled by the E2E layer. The expected value is 1, indicating that the E2E layer can successfully handle the chosen block pace.

A value for $\tau>1$ indicates that the duration of the user perception $P(k)$ measured at the client side is greater than the block duration $D(k)$. Thus, the block duration defined during the acquisition cannot be handled by the E2E layer. The case where $\tau<1$ indicates that the blocks are perceived by the user at a pace higher than the one at which they have been produced. This is only possible when the blocks are first hindered or stored for some amount of time and then sent at a faster pace.

\subsubsection{Block size ratio, $\varepsilon$ metrics}

The block size ratio $\varepsilon(k)$ related to the block $k$ is the ratio between the successfully rendered block size $S_{\mathrm{C}}(k)$ and the original block size $S(k)$ (Fig. 3).

The block size ratio can be written as

$\varepsilon(k)=\frac{S_{\mathrm{C}}(k)}{S(k)}$

The $\varepsilon$ metrics indicates if the E2E layer can successfully handle the chosen block size. If $\varepsilon<1$ part of the block was lost along the E2E transmission path either during the network transmission or at the client side if the client application cannot handle the incoming flow. The underlying network protocol can guarantee whether or not the packets are delivered. In the former case, such as in TCP, it can be assumed that the block size ratio is always 1 provided that the client communication component waits long enough to receive the complete block and that the client application has enough resources to process the received block. In the latter case, without guaranteed delivery, such as in UDP, information can be lost during the transmission. An information loss can either be due to a transmission problem, a lack of resource in the client application, or a client application deadline enforcing mechanism that stops the current block rendering when a new block becomes available.

Various measures can be taken by the client application when the current block cannot be fully handled by the E2E infrastructure. On client devices with limited resources such as PDA, the metrics are simply sent back to the server. The server takes care of adapting the block characteristics without the help of the client component to compensate for the deficient block handling, thus compensating the lack of resource/feature on the client device. This adaptation scheme is described in the next section.

The above metrics definitions used to estimate the remote experimentation QoS are somehow analogous to the measurements defining the QoS in the context of network transmission that is generally defined by a combination of bandwidth, throughput, packets jitter, packet delays and packet loss. Besides working at different abstraction level, the main difference lies in the implicit relation to the E2E infrastructure ability to sustain the incoming flow of information. The packet jitter could be compared to the block period ratio, the former giving an absolute measurement and the latter informing about the relative adequation between the generated and the perceived block pace. The block size ratio is analogous to the packet loss, the former being relative to the original block size. The quotient of the block size by the block duration defines a throughput at the application level that is analogous to the throughput at the network level. This comparison only holds if the E2E infrastructure is insensitive to the fact that the throughput is a ratio. This means that, any of the block size/duration pairs giving identical throughput would be handled similarly by the E2E infrastructure. While this conjecture can be assumed true in the case of network transmission, at the application level, the E2E infrastructure is nevertheless sensitive to the block size and duration, and therefore this would not hold.

The proposed metrics combined with the block definition are sufficient to fully characterize the E2E infrastructure, this independently of the chosen transmission protocol or transmission infrastructure.

\section{End-to-end adaptation}

The end-to-end adaptation scheme is meant to permit the user to appropriately perceive at distance the dynamics and the state of the physical equipment in a timely manner by providing the user with a QoS suitable for remote experimentation.

The end-to-end adaptation scheme is devised in two steps. First an equivalent mathematical representation for 
the E2E infrastructure and a model for the end-to-end block round-trip time are defined. Then, based on this model, a adaptation scheme is developed to track a given reference value for the block round-trip time and to adapt, in realtime, to the E2E infrastructure and to its variations.

The proposed adaptation scheme is developed to efficiently enforce the desired QoS and to maximize the use of the E2E infrastructure. The model is deterministic and based on a mass-balance conservation equivalence, which is also widely used in the process control community. Stochastic models are avoided, even though they are extensively studied in the network communication community. These models generally consider the E2E transmission at the network level without capturing the client and server behaviors at the application level [34, 35]. Moreover, they generally rely on measurements (TCP stack info, sshtresh, rtt, etc.) that are not ubiquitously available at the application level [36, 37].

By considering block as described previously, it is possible to work at a higher abstraction level. This allows the construction of a model for E2E adaptation. The proposed metrics are used to identify the model parameters and to adapt, in real-time, the sending behavior.

\subsection{E2E equivalent mathematical representations}

End-to-end equivalent mathematical representations that characterize the E2E layer at a macroscopic level are used to build an E2E model suitable for adaptation. This equivalent mathematical model is defined by three components, namely an equivalent buffer, an equivalent delay, and an equivalent bandwidth.

The equivalent E2E buffer emulates all the buffers located along the E2E path. It includes buffers found in the following three components-transmission, client, and server. The transmission-component equivalent buffer is made of the routers found along network links. Since the transmission link is perceived by the transmission component through the network APIs, a buffer related to the chosen transmission protocol might also be present (i.e. for TCP [38]). Buffers found in the client and the server components can be the result of synchronization mechanisms [22], or due to internal queues (i.e. routers queues policy). Whilst the client and server component buffers can easily be controlled or even cancelled, the equivalent buffers for the protocol and network are out of the E2E applications direct control. The only alternative is to refrain from sending additional blocks in order not to increase the buffer content. The FIFO policy observed by buffers enforces that all the remaining blocks, or part of them, found in the equivalent E2E buffer are to be emptied before the current block can be processed. The resulting effect is an added delay that degrades the interaction and should therefore be avoided. Ideally, this equivalent E2E buffer is empty, so that no time is spent to send the remaining information found in the buffer prior to the current data.

The E2E equivalent bandwidth encompasses the overall infrastructure. This is different from the network available bandwidth [39, 40] that considers only the transmission links. The E2E bandwidth $B_{\mathrm{e}}(k)$ that can be used is the minimum of the bandwidth of the various components including the server bandwidth $\left(B_{\mathrm{S}}\right)$, the client bandwidth $\left(B_{\mathrm{C}}\right)$, and the network bandwidth $\left(B_{\mathrm{N}}\right)$.

$B_{\mathrm{e}}=\min \left(B_{\mathrm{S}}, B_{\mathrm{C}}, B_{\mathrm{N}}\right)$

The block E2E equivalent propagation delay $C(k)$ represents the time for the block $k$ to actually transit from one end to the other. It is the sum of all the propagation delays found in the E2E transmission. It cannot be avoided, thus every block, whatever its size, has to undergo a duration of $C(k)$.

The propagation delays found in the server and in the client components are also included within $C(k)$. The delays representing the steady state value of the time spent within the buffers are also incorporated in $C(k)$. Also included in $C(k)$ is the time for the block acknowledgment to go from the client back to the server (see Fig. 3).

The longer the propagation delay $C(k)$, the bigger the number of blocks in transit. Similarly to the propagation delay $C(k)$, the larger the E2E bandwidth $B_{\mathrm{e}}(k)$, the bigger the number of blocks in transit.

\subsection{Model for the E2E round-trip time}

Based on the above equivalent E2E representations, a model for the end-to-end block round-trip time $d$ is proposed. This model is used for two purposes. First, it permits to develop an adaptation scheme, that efficiently tracks the block round-trip $d$ and that handles, in real-time, the E2E infrastructure variations. Second, the model is used to find a reference value that maximizes the use of the E2E infrastructure.

The model for the round-trip time is composed of a fixed part, the equivalent E2E propagation delay and a variable part represented by the time spent in the equivalent E2E buffer.

The following assumptions are made.

- The equivalent propagation delay $C(k)$ is constant. ${ }^{1}$

- The equivalent bandwidth $B_{\mathrm{e}}(k)$ is constant, ${ }^{1}$ i.e. the blocks are "traveling" within the E2E infrastructure using the same constant bandwidth $B_{\mathrm{e}}$.

\footnotetext{
${ }^{1}$ Otherwise varies slowly comparing to the adaptation time scale.
} 
- The TCP protocol is used for the transmission, thus there is no loss during the E2E transmission. In other words, blocks are fully received and perceived by the user, i.e. $\varepsilon=1$.

- The capacity of the equivalent buffer is unlimited, but it cannot be negative.

- We consider that the equipment dynamics will not change during the time of the experiment. As a consequence, the pace at which the state of the distant equipment should be and is captured remains constant. In other words, the block duration $D$ remains constant. Hence, only the block size $S(k)$ is to be modified by the adaptation mechanism.

Figure 4 represents a snapshot of the E2E infrastructure used to describe the model for block round-trip time using the mass-balance equivalence. On the left, the server process generates blocks that are passed to the E2E buffer. The server throughput is chosen such that the quantity of information $S(k)$ is fed into the equivalent buffer during a time $D$. If this is not the case, a block can be partially stored in the E2E buffer. Then, the E2E process extracts blocks from the buffer and transfers them to the other end of the equivalent pipe. The block exits the infrastructure when the server has processed the acknowledgment. The length of the pipe represents the propagation delay and the time for the last block to exit the E2E infrastructure. The time spent in the buffer has to be added to the time spent in the pipe to get the block round-trip time.

On the right of the pipe, the block $k-r$ exits the E2E infrastructure. The number of blocks in transit in the E2E infrastructure is represented by $r$. It takes a time $T(k-r)$ for the block $k-r$ (of size $S(k-r)$ ) to fully exit the E2E infrastructure using the available bandwidth $B_{\mathrm{e}} . T(k-r)$ is defined by

$T(k-r)=\frac{S(k-r)}{B_{\mathrm{e}}}$

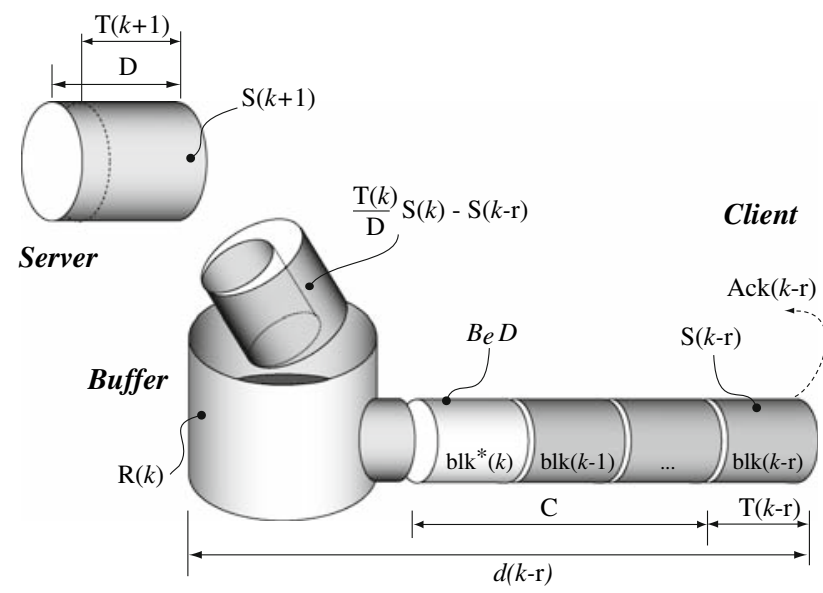

Fig. 4 Snapshot of the equivalent model for the block round-trip time considered when the block $k-r$ exits the E2E infrastructure
During its transit within the E2E infrastructure, the block $k-r$ spends time in three locations, namely, the time spent in the equivalent E2E buffer, the block propagation delay, and the time $T(k-r)$ for the block to exit the E2E infrastructure.

The time spent in the E2E buffer is function of the amount of data $R(k-r)$ that was previously stored in this buffer. If the E2E buffer is empty, i.e. $R(k-r)=0$, no time is spent in the buffer. If there are remaining data in the buffer, i.e. $R(k-r)>0$, the time spent in the buffer is given by the ratio between the buffer size $R(k-r)$ and the constant bandwidth $B_{\mathrm{e}}$. This bandwidth is identical to the bandwidth used by the E2E block extracting process.

The block propagation delay $C$ is constant. As stated earlier, the time for the acknowledgment to come back to the server is included within the propagation delay. Therefore, the block round-trip time $d(k-r)$, which is equivalent to the time for the block $k-r$ to transit within the E2E infrastructure, is given by

$d(k-r)=\frac{R(k-r)}{B_{\mathrm{e}}}+C+T(k-r)$

The dynamics of the E2E buffer are given by the previous buffer state and by the balance between the amount of data that exits the buffer and enters the buffer during the same period. The buffer is considered during the period $T(k-r)$ defined by the time for the block $k-r$ to exit the E2E infrastructure. According to the definition (4), the amount of data that exits the buffer during the time $T(k-r)$ is $S(k-r)$.

During the same time $T(k-r)$, blocks or a fraction of a block enter the E2E buffer (see the right part of Fig. 4). Given the bandwidth $B_{\mathrm{e}}$, the fraction of a block that enters the E2E infrastructure during the time $T(k-r)$ is defined by the time ratio between $T(k-r)$ and the constant block duration $D$. The state evolution of the E2E buffer without the buffer saturation, $R^{*}(k)$, is given by

$\underbrace{R^{*}(k+1)}_{\text {newstate }}=\underbrace{R^{*}(k)}_{\text {oldstate }}+\underbrace{\frac{S(k) T(k-\mathrm{r})}{D}}_{\text {enter }}-\underbrace{S(k-\mathrm{r})}_{\text {exit }}$

Shifting everything by $r$ and replacing $T(k-r)$ in (7) by its definition (4) gives

$R^{*}(k-r+1)=R^{*}(k-r)+\frac{S(k-r) S(k-2 r)}{B_{\mathrm{e}} D}-S(k-2 r)$

The E2E buffer obviously cannot have a negative value, thus (8) becomes

$R(k-r+1)=\max \left[0, R^{*}(k-r+1)\right]$

The state of the equivalent E2E buffer is expressed in bytes. This is the consequence of two factors. First, the 
balance between the amount of data that enters the buffer and the amount of data that exits the buffer may be fraction of a block. Second, the size of the blocks varies over time. Consequently, expressing the buffer state in blocks would require the complete block size history that is not available.

\subsection{E2E adaptation scheme}

The main objective of the E2E adaptation scheme is to maximize the QoS in terms of the defined metrics [29]. According to the assumptions previously made, the block round-trip time is the chosen value to be controlled and the block size is the value that is adapted (actuator). The other objectives specify that the block should be fully perceived at the same pace at which it has been acquired.

The E2E adaptation scheme relies on the values of the E2E bandwidth $B_{\mathrm{e}}$ and the propagation delay $C$ that can be estimated using the model and the metrics defined previously. The block duration $D$ and the estimated values of $B_{\mathrm{e}}$ and $C$ are necessary to compute the reference value for the block round-trip time $d_{\mathrm{r}}$ that maximizes the use of the E2E infrastructure (see Sect. 5.4 for details).

The E2E adaptation scheme can be implemented as a cascade structure with an inner loop handling a TCPfriendly network transmission and an outer loop handling the application level adaptation that controls the block round-trip time (Fig. 5). The benefit of such a cascade structure is to accommodate for any inner-loop policy, i.e. transmission protocol. TCP-friendly transmissions ensure that the Internet best practices are respected [41, 42].

\subsection{Operating condition}

The reference value for the round-trip time $d_{\mathrm{r}}$ is either defined manually by the user or computed using the block duration $D$ and the estimated values of $B_{\mathrm{e}}$ and $C$. Reference

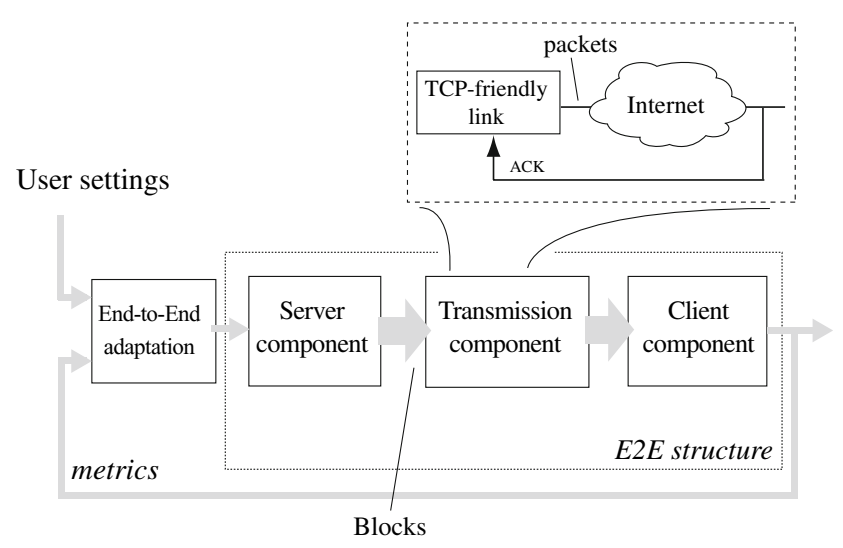

Fig. 5 The cascade structure of the E2E adaptation scheme values defined by the user may lead to instability or nonoptimal usage of the available E2E bandwidth. Hence, this option is not considered thereafter. The best-suited $d_{\mathrm{r}}$ to be computed is defined as the one that permits to fully utilize the available E2E bandwidth $B_{\mathrm{e}}$ without filling up the equivalent E2E buffer. The block size $S(k)$ is chosen accordingly. Thus, the equation for the block round-trip time $d(k)$ is given by setting the buffer size $R(k)$ to 0 in (6) and by replacing $T(k)$ by its definition (5). This finally gives

$d(k)=C+\frac{S(k)}{B_{\mathrm{e}}}$

Figure 6 represents a snapshot of the model for the block round-trip time considered when the equivalent buffer $R(k)=0$. The two black arrows on the left of Fig. 6 illustrates the adaptation of the block size $S(k+1)$.

By definition, the maximum block size $S_{\max }$ that can be handled by the E2E bandwidth $B_{\mathrm{e}}$ during a period equivalent to the block duration $D$ is given by

$S_{\max }=B_{\mathrm{e}} D$

Sending, at a pace equivalent to the constant block duration $D$, a block of size bigger than $S_{\max }$ would either result in a partial block loss $(\varepsilon<1)$ or partial block buffering $(R>0$, $\tau>1$ ), the effects of which should be avoided.

The reference round-trip time $d_{\mathrm{r}}$ represents the roundtrip time for a block of size $S_{\max }$. It is given by setting $S(k)$ to $S_{\max }$ in (10).

This gives

$d_{\mathrm{r}}=C+\frac{S_{\max }}{B_{\mathrm{e}}}$

By definition (11), $D$ is equal to $S_{\max } / B_{\mathrm{e}}$. Therefore, the above equation (12) can be rewritten as

$d_{\mathrm{r}}=C+D$

The reference round-trip time $d_{\mathrm{r}}$ is equivalent to the block duration $D$ plus the E2E propagation delay $C$. This reflects

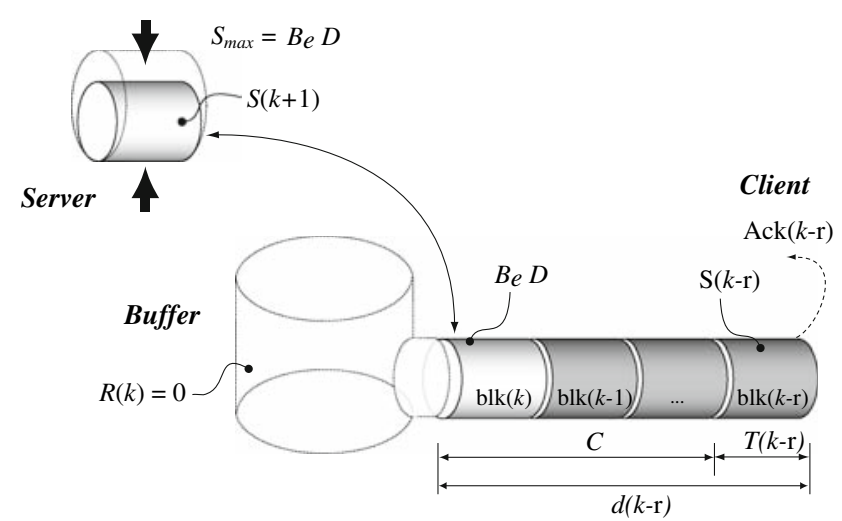

Fig. 6 E2E infrastructure representation when the E2E buffer is empty $R(k)=0$ 
the objective of choosing a $S_{\max }$ that maximizes the use the E2E transmission path. In other words, $d_{\mathrm{r}}$ specifies the block size that would fill the portion of the pipe (as portrayed in Fig. 6) which represents the block duration $D$. Note that the above chosen $d_{\mathrm{r}}$ implies that there is at least one block in transit.

For any $d_{\mathrm{r}}$ smaller than the one defined above, the pipe (Fig. 6) will only be partially filled up. To the contrary, with $d_{\mathrm{r}}$ larger than the one defined above, part of the block will remain in the equivalent $\mathrm{E} 2 \mathrm{E}$ buffer and triggers oscillations in the block round-trip time, something that has to be avoided.

The above discussion clearly shows the advantage of having an equivalent model for the block round-trip time. It allows to directly choosing the appropriate value for the block round-trip time reference. Also, it avoids selecting a value that either under-utilizes or over-utilizes the E2E resource.

\subsection{The controller}

The role of the adaptation scheme is threefold. First, the controller tracks the block round-trip time. Second, using the model defined previously, it estimates the E2E bandwidth $B_{\mathrm{e}}$ and the propagation delay $C$. Third, based on the block duration $D$ and the estimated $B_{\mathrm{e}}$ and $C$, it computes according to the operating condition (O.C.) the reference value for the block round-trip time $d_{\mathrm{r}}$ that maximizes the use of the E2E infrastructure (Fig. 7).

The controller that tracks the reference block round-trip time $d_{\mathrm{r}}$ by rejecting the fast disturbances in the underlying E2E infrastructure. These disturbances may be the result of transmission link variation such as the increase of distance between the access point and the client device in a wireless network or due to an increase in the transmission link cross traffic. Disturbances may also be the result of a temporary lack of resource at the client side. In this last case, the E2E buffer will fill-up.

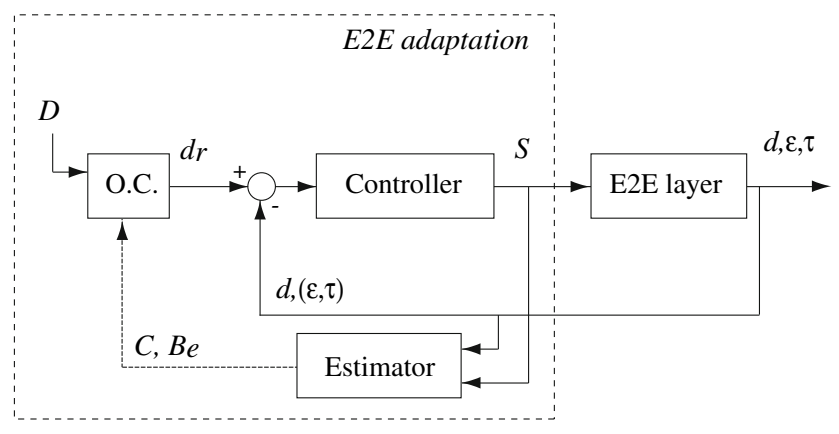

Fig. 7 The E2E adaptation scheme
The normal operating condition defines that the E2E buffer is empty and sees non-empty buffer as a disturbance. Thus, the dynamics of the E2E infrastructure is given by the first mode of the block round-trip time model when $R(k)=0$. The chosen controller is an integral controller. The integral controller has the advantage of approaching smoothly the reference value according to its integral gain. Another benefit is the low-pass filtering effect that smoothes the potential noise added to the block round-trip time measurement. An anti-reset windup ARW mechanism should be added to limit the internal dynamics of the controller [43].

The values $C$ and $B_{\mathrm{e}}$ are estimated by using linear leastsquare method. To guarantee that the least-square estimation is correct, it is primordial to guarantee that $\mathrm{S}(k)$ and $\mathrm{d}(k)$ are measured when the equivalent E2E buffer is empty. Otherwise (10), used for the least-square estimation, is incorrect and the estimation returns erroneous results. The E2E bandwidth $B_{\mathrm{e}}$ and the $\mathrm{E} 2 \mathrm{E}$ propagation delay $C$ are estimated continuously but the values used to compute $d_{\mathrm{r}}$ should be updated only when a significant variation is detected.

\section{Application}

This section presents an example of ubiquitous remote experimentation, where the physical equipment to be remotely controlled corresponds to the one used by students at the Ecole Polytechnique Fédérale de Lausanne (EPFL). The whole framework is used to carry out remote hands-on laboratory assignments in automatic control [44]. The standard client and server application (written in LabVIEW), does not have a real-time E2E adaptation mechanism. As a consequence, the server, which uses the UDP protocol, tends to saturate the available bandwidth. Also, the client application has a simple built-in mechanism that drops information whenever it cannot properly process it, which results in a waste of both server and transmission resources. Therefore, the current server setting has been modified to implement the proposed adaptation scheme and a light PDA client has been written accordingly. Moreover, the UDP protocol has also been replaced by the TCP protocol so as to ensure friendliness among transmissions.

The real-world results presented hereafter show how the adaptation scheme adjusts itself to the E2E infrastructure characteristics, especially those of the network and client. To convincingly illustrate the benefit of the proposed E2E adaptation scheme, the E2E infrastructure has been selected such that it can easily be saturated by the amount of transmitted information and can also be externally perturbed. 
Figure 8 depicts the chosen E2E infrastructure. It consists of a palm handheld device (PDA) with limited resources (the client) connected to a desktop computer (the server). The information transits wirelessly (Bluetooth) from the handheld device to the server using the TCP protocol, thus the $\varepsilon(k)$ metrics (block size ratio) is always equal to 1 .

The chosen equipment to be remotely controlled is a laboratory-scale electrical drive with a rotating load. It is connected to the server through a data acquisition board. The experimentation protocol for the students consists in choosing the right set of parameters to dynamically position the rotating load while fulfilling some specifications (overshot, settling time, etc.). This is a typical example used in many textbooks to illustrate automatic control theory. The load motion is captured by a video camera. Remote experimentation of such mechatronic devices has strong real-time constraints due to the fast dynamical behavior of the equipment to be observed and controlled. In such a case, blocks should be updated at least 4 times per second to adequately capture the state of the distant equipment, i.e. the load motion. This gives a block duration of $D=250 \mathrm{~ms}$.

As proposed previously, a pure integral controller is implemented at the server side to track the block round-trip time $d_{\mathrm{r}}$. To achieve this specification, the integral controller (I) adjusts in real-time the block size $S(k+1)$ based on the measured $d(k)$ and the reference block round-trip time $d_{\mathrm{r}}$ (Fig. 9). An anti-reset windup mechanism is also implemented.

The values for $B_{\mathrm{e}}$ and $C$ are estimated using a leastsquare technique based on the measured $d(k)$ and $S(k)$ of the first few blocks sent. These blocks are specially crafted

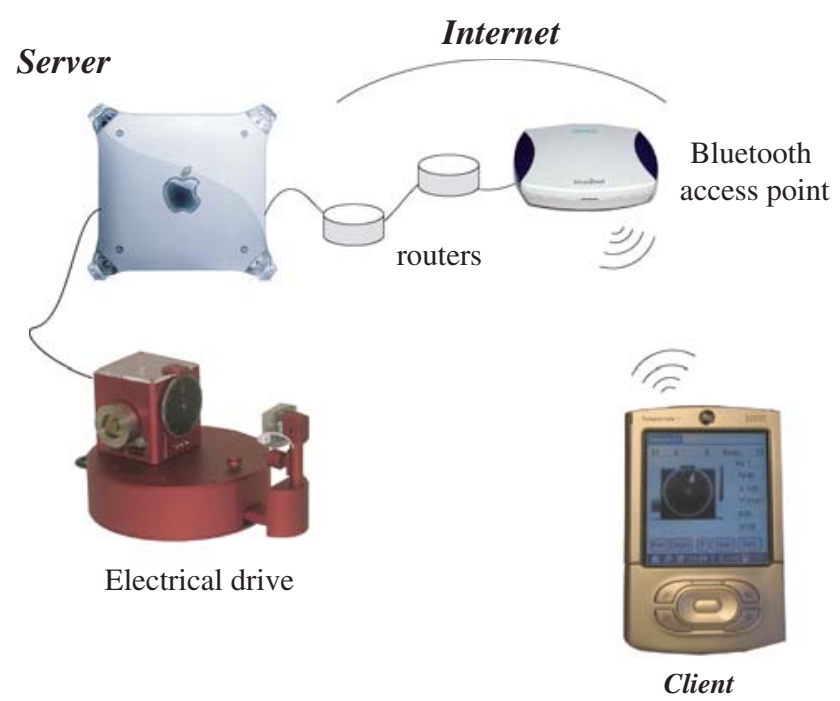

Fig. 8 The chosen E2E infrastructure includes a PDA connected wirelessly to the remote server to ensure a valid estimation, i.e. the equivalent E2E buffer remains empty. Based on the identified $B_{\mathrm{e}}(15.8 \mathrm{kB} / \mathrm{s})$ and $C(110 \mathrm{~ms})$ and using the defined block period $D(250 \mathrm{~ms})$, the reference value for the block round-trip time is $d_{\mathrm{r}}=$ $360 \mathrm{~ms}$. This value of $d_{\mathrm{r}}$ maximizes the usage of the considered E2E infrastructure.

Figure 10 shows the impact of the adaptation scheme with the controller activated after about 100 blocks of minimal size data have been sent. At that time, the block round-trip time $d(k)$ (Fig. 10 left) increases until the computed set point $d_{\mathrm{r}}$ is reached. As a result, the block size $S(k)$ increases until $S_{\max }$ is reached (Fig. 10 right) upon which the E2E infrastructure is fully utilized for the given block duration $D$.

Figures 11 and 12 show the cases where a non-optimal reference value $d_{\mathrm{r} 2}$ has been chosen for the block round-trip time. In Fig. 11, the chosen reference value $d_{\mathrm{r} 2}$ is smaller than the optimal one $d_{\mathrm{r} 1}$. To reach $d_{\mathrm{r} 2}$, the controller reduces the block size $S(k)$ to a value smaller than the

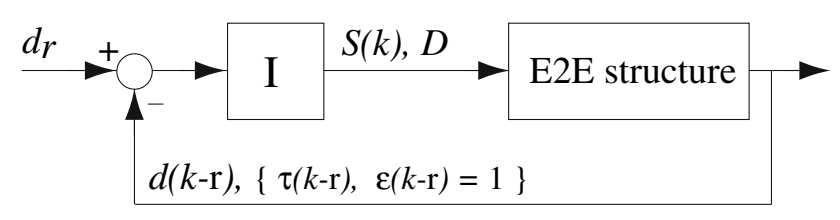

Fig. 9 E2E adaptation scheme for tracking $d$
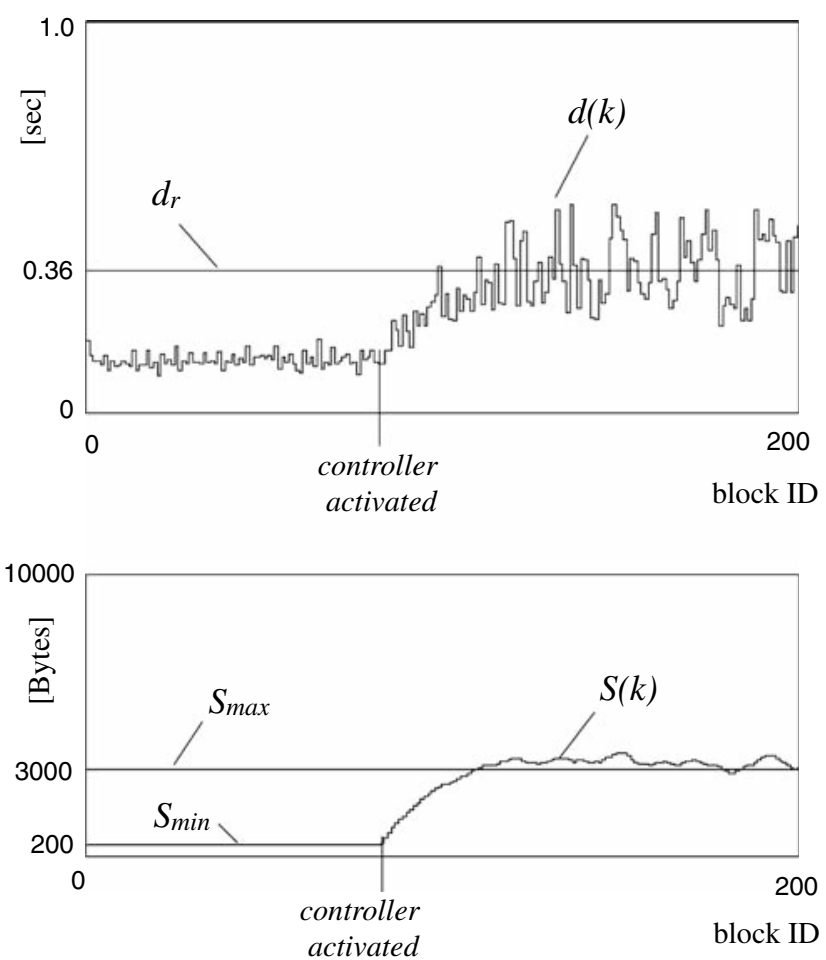

Fig. 10 Evolution of the block round-trip times and sizes before and after the activation of the controller 

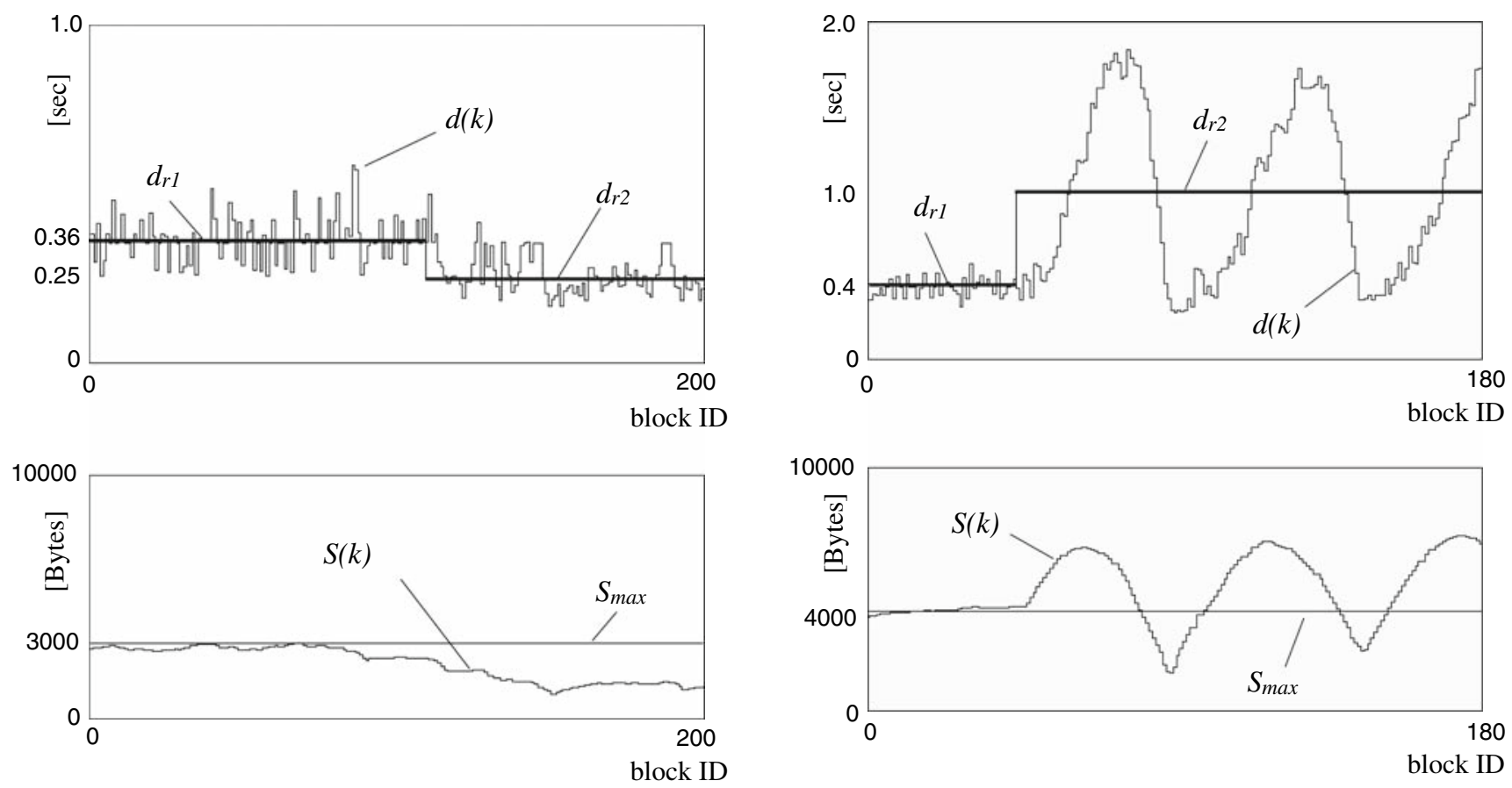

Fig. 11 Measured $d(k)$ (left) and block sizes evolution (right) when where the reference changes from the optimal value $d_{r 1}$ to the nonoptimal one $d_{r 2}$

optimal $S_{\max }$. As a result, the E2E infrastructure usage is not maximized.

The block size cannot be smaller than $S_{\text {min }}$, which correspond to the smallest possible packet containing only the information needed for the metrics estimation. Also, $d_{\mathrm{r} 2}$ should obviously not be set smaller than the E2E propagation delay $C$.

In Fig. 12, the chosen reference value for the block round-trip time $d_{\mathrm{r} 2}$ is larger than the computed value $d_{\mathrm{r} 1}$. The E2E controller tracks this new value by increasing the block size $S(k)$ until it reaches $d_{\mathrm{r} 2}$. Since the E2E bandwidth $B_{\mathrm{e}}$ remains unchanged, the E2E infrastructure cannot accept a block bigger than $S_{\max }$. Thus, blocks are stored in the E2E buffers $R(k)$. Consequently, the block round-trip time $d(k)$ increases. To compensate for this increase the controller reduce the block size causing unwanted oscillations in both block sizes and block round-trip times.

These results clearly show the importance of having a valid model to define the reference value for the block round-trip time $d_{\mathrm{r}}$. A reference value smaller than the optimal one does not use all the available E2E capacity, so as to maintain the system stable. On the other hand, a reference value larger than the optimal value leads to unwanted oscillations in both block sizes and block roundtrip time, which should definitely be avoided through automatically rejecting these values.

The next results illustrate the ability of the proposed E2E adaptation scheme of rejecting disturbances. These

Fig. 12 The measured $d(k)$ (left) and lock sizes evolution (right) when the reference value changes from the computed $d_{r l}$ to the flawed $d_{r 2}$

disturbances are generated by rapidly changing the characteristics of the E2E infrastructure. This is done by moving the PDA away from the access point, hence producing a large bandwidth variation. Similar disturbances could also be produced either by generating a large amount of cross-traffic or by limiting resources at the client side.

Figure 13 shows the block round-trip time $d(k)$ variations resulting from the PDA displacement. Before time $t_{1}$, the PDA is close to the access point. Then, at time $t_{1}$, the PDA is rapidly placed at a distance of about $10 \mathrm{~m}$ from the access point and it stays there until time $t_{2}$. At time $t_{2}$, the PDA is brought back to its original position.

The disturbances applied to the E2E infrastructure is significant. At the application level, the largest measured block round-trip time is about $5 \mathrm{~s}$, more than ten times the initial block round-trip time. To reject this disturbance, the controller quickly reduces the block size $S(k)$ in order not to worsen the situation. Since the controller cannot send blocks of a negative size, so as to empty the E2E buffer, it sends blocks of a minimal size $S_{\min }$ until $d(k)$ crosses $d_{\mathrm{r}}$ (remember that blocks must be sent to estimate metrics). At that time, the controller starts to increase the block size until a new equilibrium point resulting from the new infrastructure characteristics is reached. This new value is smaller than the original $S_{\max }$.

At time $t_{2}$, the PDA is brought back to its original position. The controller increases the block size so as to compensate the E2E characteristic variation. Note that the 

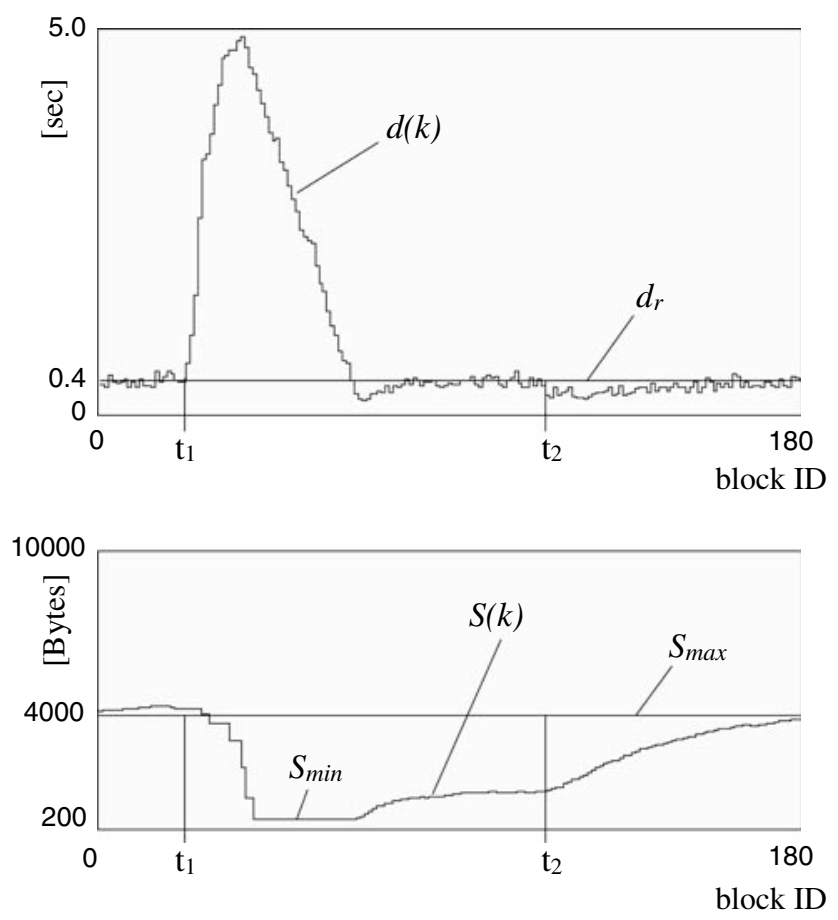

Fig. 13 The measured block round-trip time $d(k)$ and block size $S(k)$ subject to disturbances. At time $t_{1}$, the PDA is rapidly placed at a distance; at time $t_{2}$ it is brought back to its original position

buffer is not needed, and therefore there is no buffer delay in the controller response, but only a slight undershoot in $d(k)$.

Figure 14 shows the evolution of the $\tau(k)$ metrics. When the disturbance occurs at time $t_{1}$, the blocks are available to the user with some delay due to the buffering effect. This results resulting $\tau(k)>1$. Then, when the buffer drains, the blocks arrive at a pace faster than the block duration, so that $\tau(k)<1$. Due to the limited resources on the PDA, no mechanism is implemented so as to compensate for the jitter introduced by the disturbance; the blocks are played as they arrive.

The above experiments show that the proposed E2E adaptation scheme can successfully adapt to the available E2E infrastructure and reject disturbances while relying

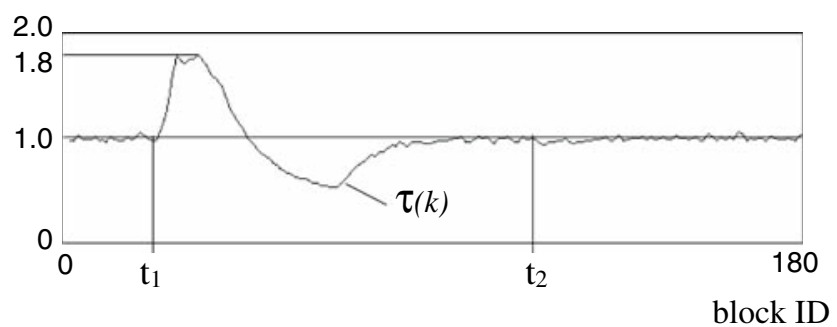

Fig. 14 The $\tau$ metrics variation resulting from the applied disturbance only measurements made at the application level. It does not depend specifically on the underlying type of connection, be it the protocol or the client device. Only the E2E metrics are estimated and used by the controller.

The effect of the above disturbance applied to the E2E infrastructure has also been observed at the network level. To achieve this, a packet capture program (tcpdump) is used to record packets (not blocks) as they leave the computer. In parallel, the server application has been instrumented to record the block information $S(k)$ and $T_{\mathrm{S}}(k)$. The server generates blocks at a regular pace $D$. Then, within the transmission component, the chosen protocol, TCP, splits blocks into packets and sends theses packets according to its own set of rules [45, 46]. Each TCP packet carries a unique identifier called the sequence ID. When packets leave the computer, the TCP increments the packet sequence ID by a value corresponding to the packet size. The packet capture program records these sequences of ID values. Block sizes are recorded, and therefore block sequence IDs representing the accumulated block size can be computed and compared to the TCP packet sequence IDs. By comparing the two recorded ID sequences, it is possible to determine whenever a given block (split into packets) has left entirely the computer. A block has left the computer whenever the TCP sequence ID is the same as the block sequence ID (i.e. the same $y$-coordinate). TCP has a built-in mechanism that adapts to the available network bandwidth [47]. This internal mechanism buffers data that cannot be immediately sent. At the considered application level, there are no direct means to measure the state of a TCP connection.

The upper part of Fig. 15 shows two curves; one corresponds to the TCP sequence IDs vs. time; the other corresponds to the accumulated blocks size versus time. Prior to the time $t_{1}$, the server generates blocks of similar sizes at a fixed pace $D$. Consequently, the block slope is constant. The corresponding TCP sequence-ID curves closely follow the block curves indicating that TCP packets leave the computer without further delay. The curves overlay shows that the controller successfully sets the block size to a value that matches the E2E infrastructure characteristics. When the user moves away (time $t_{1}$ ), the slope of the TCP curve flattens considerably indicating that the TCP adapts to the new network condition. At that instant of time, the server has not reduced the block size, and therefore blocks (that cannot be sent) are delayed (buffered) by TCP. This delay can be measured on the yaxis. The controller reacts to this increase in the block round-trip time by quickly reducing the block size to its minimal value. As a result, the block curves flatten considerably. The block curve remains almost horizontal until the TCP curve rejoins the block curve indicating that blocks are no longer delayed nor buffered. Then, the 


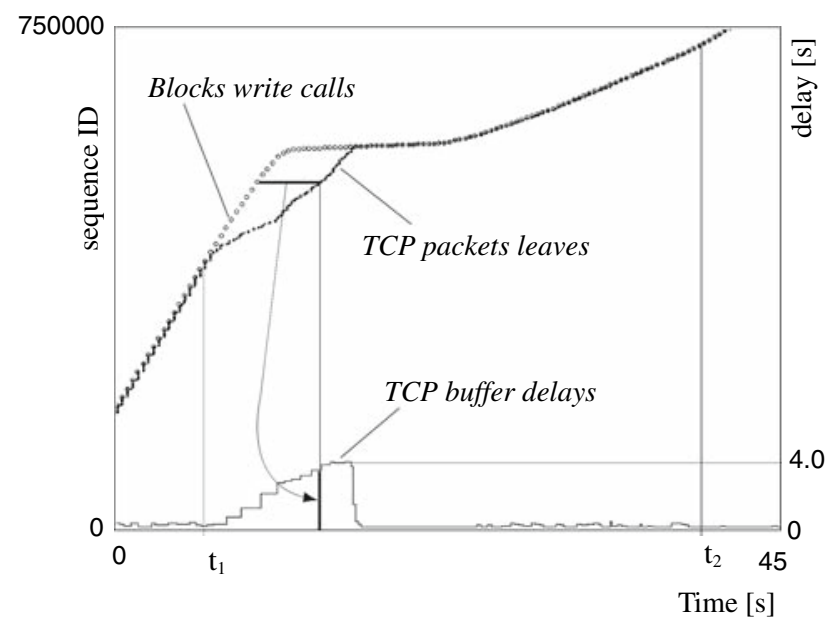

Fig. 15 Effect of the disturbance applied to the E2E infrastructure observed at the network level

controller increases the block size until the second steady state value is reached. When the user comes back to its original position (time $t_{2}$ ), the controller adapts to the new E2E characteristics by increasing the block size. As a consequence, the slope of both the block and the TCP curves increase back to their initial value.

The lower part of Fig. 15 shows the time spent in the TCP internal buffer that corresponds to the difference between the block and the TCP curves along the $x$-axis. Note that the TCP buffer delay curve is similar to the block round-trip time $d(k)$ in Fig. 13 which indicates that, for this disturbance, the TCP buffering effect is the main contributor to the E2E delay.

The controller is able to successfully track the reference value $d_{\mathrm{r}}$ when the E2E characteristics are significantly modified. After the transient phase, the controller rejects the remaining constant disturbance. In other words, the E2E adaptation scheme successfully guarantees the QoS, i.e. the interaction, when a disturbance is applied to the E2E infrastructure, this occurs independently of either the underlying infrastructure or the transmission protocol or the client device.

\section{Conclusion and future work}

Remote experimentation over the Internet is nowadays common for users and especially students working over wired networks. This is not the case for mobile users using lightweight devices with limited resources. The rapid variations of the wireless communications characteristics and the reduced performance of the client devices may severely degrade the user experience if the proposed remote experimentation solution does not adapt to these new constraints.
This paper presents a new end-to-end adaptation scheme to provide the user with the best possible interacting experience, while considering imposed constraints in the ubiquity of the solution, the adaptability to the E2E infrastructure, and the respect of the Internet best practice. It is applied in flexible engineering education to promote and develop ubiquitous and mobile learning opportunities.

The proposed scheme first specifies the objective of providing an adequate user experience in terms of QoS from usability and educational points of view. It offers a responsive feedback to a user action while guaranteeing that the drawbacks inherent to the distance between the user and the physical equipment are minimized.

This objective has been formalized by three properties that need to be satisfied in order to provide a suitable user experience, namely the level of interaction which represents how quickly a feedback is provided to the user, the system dynamics rendering which represents how accurately in time the behavior of the remote system is perceived and the amount of semantic content that represents how well the distant equipment state and conditions of operation can be perceived by the client. These three properties define the quality of service for ubiquitous remote experimentation.

There is no sensor that can directly measure the proposed QoS. Therefore, three metrics are proposed to estimate the QoS. The definition of the metrics relies on two abstractions. The first one defines a block as a unit of information that fully describes the state and the conditions of operation of the remote equipment at a given time. The second abstraction defines an end-to-end infrastructure that includes the communication link and both the server and the client devices at the application level. The estimated metrics are the block end-to-end round-trip time, the blockperiod ratio and the block-size ratio. The proposed metrics combined with the block definition are sufficient to characterize the capabilities of the end-to-end infrastructure.

To guarantee a given QoS to the distant user, an adaptation scheme is proposed. This scheme is implemented as a cascade structure, where the outer loop controls the block round-trip time and the inner one ensures a TCP-friendly transmission. Thanks to this cascade structure, the adaptation scheme is independent of the chosen inner-loop policy.

The end-to-end infrastructure can be represented as an equivalent buffer, an equivalent propagation delay and an equivalent bandwidth. The main objective for ubiquitous remote experimentation is level of interaction that can be measured by the block round-trip time. A model for the block round-trip time has been derived from the equivalent representations. This model allows not only the design of an adaptation scheme that successfully tracks the block round-trip time, but also enables to reject, in 
real-time, disturbances due to the variations of the E2E infrastructure characteristics. Based on this model, it is also possible to specify a reference value for the block round-trip time that ensures the full utilization of the E2E infrastructure.

The proposed scheme has been successfully implemented to control a laboratory-scale electrical drive from a PDA handheld computer. The chosen transmission media was a Bluetooth connection that could easily be disturbed. The end-to-end adaptation scheme implemented on the top of TCP connection successfully adapted to the end-to-end infrastructure and the induced disturbances were successfully rejected.

The illustrative application clearly shows that it is possible to successfully control, at the application level, the E2E round-trip time even if the chosen transmission protocol is TCP. This allows for effective remote experimentation.

Using this adaptation scheme, ubiquitous remote experimentation activities are now possible in engineering education as a complement to common remote experimentation activities. This new paradigm will be proposed to students at the EPFL starting from 2008 [48].

This paper proposes a broad albeit consistent foundation for studying real-time interaction in the context of remote experimentation. It is built around two main contributions. First, based on the identified objectives of a successful interaction, the quality of service for remote experimentation is defined. The associated metrics and abstractions, which allow working at the right level of abstraction, are also defined. Second, using the provided metrics and abstractions, an adaptation scheme that enforces the QoS provided to the user is proposed and successfully validated. This scheme can be extended to consider the multivariable case where not only the block size is modified but also the block pace. This QoS can also be extended to consider the user quality of perception of the block content. This extended scheme would require a model of the user perception; this is a difficult task that requires offline identification.

\section{References}

1. Gillet D, Nguyen AV, Rekik Y (2005) Collaborative web-based experimentation in flexible engineering education. IEEE Trans Educ Spec Issue Web-based Instr 48(4):696-704

2. Ko CC, Chen BM, Chen J, Zhuang Y, Tan KC (2001) Development of a web-based laboratory for control experiments on a coupled tank apparatus. IEEE Trans Educ 44(1)

3. Schmid C (2003) Remote experimentation in control engineering. In: Proceedings of the 11th Mediterranean conference on control and automation MED'03, Rhodos, Paper IV12-01

4. Dabney JB, McCune J, Ghorbel FH (2003) Web-based control of the rice SPENDULAP, Int J Eng Educ 19:478-486
5. Salzmann C, Gillet D, Huguenin P (2000) Introduction to realtime control using $\mathrm{LabVIEW}^{\mathrm{TM}}$ with an application to distance learning. Int J Eng Educ Spec Issue: LabVIEW Appl Eng Educ 16(3):255-272

6. Tzafestas CS, Palaiologou N, Alifragis M (2006) Virtual and remote robotic laboratory: comparative experimental evaluation. IEEE Trans Educ 49(3):360-369

7. Callaghan MJ, Harkin J, McGinnity TM, Maguire LP (2003) Adaptive intelligent environment for remote experimentation. In: Proceedings of the IEEE/WIC international conference on web intelligence (WI'03), p. 680

8. Cooper M (2005) Remote laboratories in teaching and learningissues impinging on widespread adoption in science and engineering education. Int J Online Eng, 1.1. http://www.i-joe.org_/ ojs_/viewarticle.php?id=11. Accessed 26 Jun 2005

9. Floyd S (2000) Congestion control principles, RFC 2914, September

10. Various authors, Internet best current practices index, online reference at: http://www.faqs.org_/rfcs_/bcp-index.html

11. Bouch A, Sasse MA, DeMeer H (2000) Of packets and people: a user-centered approach to quality of service. In: Proceedings of the IWQoS2000, pp. 189-197

12. Chalmers D, Sloman M (1999) A survey of quality of service in mobile computing environments. IEEE Communications Surveys, 2nd Quarter, IEEE CS

13. Cole RG, Rosenbluth JH (2001) Voice over IP performance monitoring. ACM SIGCOMM Comput Commun Rev 31(2)

14. Aurrecoechea C, Campbell AT, Hauw L (1998) A survey of QoS architectures. ACM/Springer Verlag Multimedia Syst J Spec Issue QoS Arch 6(3):138-151

15. Gracanin D, Zhou Y, DaSilva LA (2004) Quality of service for networked virtual environments. Commun Mag IEEE 42(4):4248

16. Anbazhagan M, Nagarajan A, Understanding quality of service for Web services. ftp://www6.software.ibm.com/software/ developer/library/ws-quality.pdf. Accessed Jan 2002

17. Zhang Q, Zhu W, Zhang Y-Q (2005) End-to-end QoS for video delivery over wireless internet. Proc IEEE 93(1)

18. Nguyen AV, Rekik Y, Gillet D (2007) Iterative design and evaluation of a web-based experimentation environment. In: Lambropoulos N, Zaphiris P (eds) The book user-centered design of online learning communities. IDEA Group Inc., Hershey, pp 286-313

19. Khamis AM, Rodriguez FJ, Salichs MA (2003) Remote interaction with mobile robots, autonomous robots, vol 15-3. Springer, The Netherlands, pp 267-281

20. MacKenzie IS, Ware C (1993) Lag as a determinant of human performance in interactive systems. In: Proceedings of the SIG$\mathrm{CHI}$ conference on human factors in computing systems. ACM Press, New York, pp 488-493

21. Borkowski S, Letessier J, Bérard F, Crowley JL (2006) Usercentric design of a vision system for interactive applications. In: Proceedings of ICVS '06, IEEE international conference on computer vision systems

22. ITU Standard G.114. Audio delay recommendations. http://www.itu.ch

23. Ando N, Lee J, Hashimoto H (1999) A study on influence of time delay in teleoperation. In: Proceeding of the 1999 IEEE/ASME international conference on advanced intelligent mechatronics, Atlanta, USA, pp 317-322

24. Nguyen T, Zakhor A (2002) Distributed video streaming with forward error correction. In: Proceedings of the packet video workshop, Pittsburgh, USA

25. Gulliver SR, Ghinea G (2004) Changing frame rate, changing satisfaction? IEEE international conference on multimedia and expo, Taipei, Taiwan, vol 1, pp 177-180 
26. Ghinea G, Chen SY (2006) Perceived quality of multimedia educational content: a cognitive style approach. In: Multimedia systems, vol 11-3. Springer, Berlin, pp 271-279

27. Verscheure O, Frossard P, Hamdi M (1999) User-oriented QoS analysis in MPEG-2 video delivery. J Real-Time Imaging Spec Issue Real-Time Digit Video Multimedia Netw 5(5):305-314

28. Floyd S, Fall K (1999) Promoting the use of end-to-end congestion control in the internet. IEEE/ACM Trans Netw 7-4:458-472

29. Abdelzaher TF, Stankovic JA, Lu C, Zhang R, Lu Y (2003) Feedback performance control in software services. IEEE Control Syst 23(3), 74-90

30. Bajic IV, Tickoo O, Balan A, Kalyanaraman S, Woods JW (2003) Integrated end-to-end buffer management and congestion control for scalable video communications. In: Proceedings of IEEE international conference on image processing (ICIP), Barcelona, Spain, vol 3, pp III-257-III-260

31. Kim J, Kim Y-G, Song H, Kuo T-Y, Chung YJ, Jay Kuo C-C (2000) TCP-friendly Internet video streaming employing variable frame-rate encoding and interpolation. IEEE Trans Circuits Syst Video Technol 10(7):1164-1177

32. Gurtov A, Floyd S (2004) Modeling wireless links for transport protocols. ACM CCR 34-2:85-96

33. Legall D (1992) The MPEG video compression algorithm. Image Commun 4:129-140

34. Padhye J, Firoiu V, Towsley D, Kurose J (2000) ModelingTCP reno performance: a simple model and its imperical validation. IEEE/ACM Trans Netw 8(2):133-145

35. Khalifa I, Trajkovic L (2004) An overview and comparison of analytical TCP models. In: IEEE International Symposium Circuits and Systems, Vancouver, vol V, pp 469-472

36. Yang M, Li XR, Chen H, Rao NSV (2004) Predicting internet end-to-end delay: an overview. In: Proceedings of the 36th IEEE southeastern symposium on systems theory, Atlanta, GA, pp 210 214
37. Jaiswal S, Iannaccone G, Diot C, Kurose J, Towsley D (2004) Inferring TCP connection characteristics through passive measurements. In: Infocom '04, vol 3, pp 1582-1592

38. Stevens WR (1994) TCP/IP illustrated, vol 1. Addison-Wesley, Reading

39. Prasad R, Dovrolis C, Murray M, Claffy K (2003) Bandwidth estimation: metrics, measurement techniques, and tools. Netw IEEE 17-6:27-35

40. Jain M, Dovrolis C, End-to-end available bandwidth: measurement methodology, dynamics, and relation with TCP throughput. In: Proceedings of ACM SIGCOMM, Pittsburg, PA, USA

41. Mahdavi J, Floyd S (1997) TCP-friendly unicast rate-based flow control. Technical note sent to the end2end-interest mailing list. http://www.psc.edu_/networking_/papers_/tcp_friendly.htm. Accessed 8 Jan 1997

42. Widmer J, Denda R, Mauve M (2001) A survey on TCP-friendly congestion control. IEEE Netw 15-3:28-37

43. Åström KJ, Hagglund T (1995) PID controllers: theory, design and tuning. Instrument Society of America, New York

44. Gillet D, Fakas G (2001) eMersion: a new paradigm for webbased training in mechanical engineering education. In: International conference on engineering education, Oslo, Norway, vol 8B4, pp 10-14

45. Allman M, Paxson V, Stevens W (1999) TCP congestion control, RFC 2581, proposed standard. ftp://ftp.isi.edu/in-notes/ rfc2581.txt

46. Chiu D-M, Jain R (1989) Analysis of the increase and decrease algorithms for congestion avoidance in computer networks. Comput Netw ISDN Syst 17

47. Jacobson V (1988) Congestion avoidance and control. In: ACM SIGCOMM '88, pp 314-329

48. eMersion portal. http://emersion.epfl.ch 